\title{
DETERMINACIÓN DE FACTORES INVOLUCRADOS EN LA VALORACIÓN DEL RIESGO POTENCIAL ASOCIADO A LA DISPERSIÓN ATMOSFÉRICA DE JALES MINEROS Y A SU INGESTIÓN
}

Determination of factors involved in the assessment of potential risk of atmospheric dispersion and ingestion of mining tailings

Irma C. GAVILÁN-GARCÍA ${ }^{1 *}$, Luis A. LADINO², Eliacim FRANCO ${ }^{1}$ y Javier JUÁREZ ${ }^{2}$

${ }^{1}$ Facultad de Química, Universidad Nacional Autónoma de México, Circuito Exterior s/n, Ciudad Universitaria, 04510 Ciudad de México, México

${ }^{2}$ Centro de Ciencias de la Atmósfera, Universidad Nacional Autónoma de México, Circuito de la Investigación Científica s/n, Ciudad Universitaria, 04510 Ciudad de México, México

*Autor para correspondencia: irmac@unam.mx

(Recibido: octubre 2018; aceptado: abril 2019)

Palabras clave: minería, arsénico, biodisponibilidad, partículas de aerosol

\section{RESUMEN}

La industria minera es una actividad próspera que ofrece beneficios económicos y oportunidades laborales importantes en los estados donde se desarrolla. Sin embargo, los residuos mineros denominados jales son de gran preocupación debido al riesgo de dispersión eólica y a la liberación de especies químicas por efectos de lixiviación y su consecuente daño a la población y a los ecosistemas. El objetivo de este trabajo fue: $a$ ) evaluar la biodisponibilidad in vitro del arsénico por ingestión de polvo de jales, mediante un método que simula las condiciones gástricas e intestinales del cuerpo humano, en el distrito minero de Zimapán, Hidalgo, México, y $b$ ) determinar la concentración de arsénico por tamaño de partícula usando espectrometría de absorción atómica acoplada a generador de hidruros. Con los resultados del estudio se pretende aportar información que permita determinar el riesgo que representan los jales mineros en la zona de estudio. Los resultados muestran que el As se libera en mayor proporción cuando el $\mathrm{pH}$ del medio es ácido, esto es, en condiciones gástricas, existiendo una disminución cuando los niveles de $\mathrm{pH}$ tienden a la neutralidad en condiciones intestinales. En lo que se refiere a los resultados de la generación de un aerosol proveniente de residuos mineros, los resultados muestran que la mayor concentración de As se presentó en partículas de $1 \mu \mathrm{m}$, lo que representa un gran riesgo para la población, ya que se encuentran dentro de la fracción respirable (i.e., $\mathrm{PM}_{2.5}$ ).

Key words: mining, arsenic, bioavailability, aerosol particles

\begin{abstract}
Even though the mining industry is a prosperous activity that offers economic benefits and important job opportunities in the states where it is developed, it generates negative and cumulative environmental impacts. The waste generated, i.e. mining tails, is the biggest concern due to the risk of wind dispersion and the release of chemical species by leaching effects and their consequent damage to the population and ecosystems. The
\end{abstract}


main objective of this work was: $a$ ) to evaluate the in vitro bioavailability of arsenic by ingestion of mine tailings dust using a method that simulates the gastric and intestinal conditions of the human body in the mining district of Zimapán, Hidalgo, Mexico, and $b)$ to determine the concentration of arsenic by its particle size using atomic absorption spectrometry coupled to hydride generator. The results of this study are intended to provide information to determine the risk that mine tailings represent to the studied area. The results show that As is released in greater proportion when the $\mathrm{pH}$ of the medium is acid in gastric conditions. However, when the $\mathrm{pH}$ levels tend to neutrality in intestinal conditions there is a decrease in the release of As. Regarding the generation of an aerosol from mining waste, the results show that the highest concentration of As was present in particles of $1.0 \mu \mathrm{m}$. This represents a high risk to the population, given these particles are in the breathable fraction (i.e., $\mathrm{PM}_{2.5}$ ).

\section{INTRODUCCIÓN}

La riqueza de los yacimientos minerales de México ha conformado un sector económico de gran importancia, que en 2018 alcanzó la cifra de 907 $980 \mathrm{mp}$ equivalentes a $4.3 \%$ del PIB (INEGI 2018). Actualmente la industria de la minería se desarrolla de manera más productiva en los estados de Sonora, Zacatecas, Chihuahua, Durango y San Luis Potosí (SGM 2016).

La minería es una actividad próspera que ofrece importantes oportunidades laborales, aunque de forma paralela genera impactos ambientales negativos y acumulativos, así como efectos sociales en comunidades que exigen prácticas responsables en sus procesos (Botín 2010).

Los residuos mineros de la flotación, que en México se denominan jales, se conocen internacionalmente como colas (tailings en inglés) y relaves. La composición química y mineralógica de los jales es variada; generalmente contienen sulfuros metálicos residuales como $\mathrm{FeS}_{2}, \mathrm{Fe} 1-\mathrm{XS}, \mathrm{PbS}, \mathrm{ZnS}, \mathrm{CuFeS}_{2}$ y FeAsS, que son fuente de elementos potencialmente tóxicos (EPT) como As, $\mathrm{Cd}, \mathrm{Pb}, \mathrm{Cu}, \mathrm{Zn}$, etc. (Romero et al. 2008), que pueden ser transportados por agua, aunque la ruta de dispersión más importante es la eólica.

Los jales mineros son altamente tóxicos para los organismos y son inhibidores de factores ecológicos (tanto físicos como químicos) que afectan el crecimiento y desarrollo de la vida en general (Puga et. al. 2006a, Lillo 2011, Bartkowiak y Lemanowicz 2014). Asimismo, los daños a la vegetación generada por los jales están muy relacionados con la presencia de sales de calcio y sodio, y con la falta de nutrimentos, especialmente el nitrógeno.

En las zonas mineras donde el clima predominante es árido o semiárido, la contaminación ocurre por dispersión eólica, y en climas húmedos generalmente se presenta dispersión hídrica del material sólido, y en forma importante como soluciones cuando hay un drenaje ácido (Pérez-Martínez y Martín-Romero 2015).

Martínez et al. (2000, citado en Moreno-Tovar et al. 2012), en su estudio del comportamiento químico de metales (Fe, Mn, $\mathrm{Pb}$ y $\mathrm{Zn}$ ) en los jales de una mina en Zimapán, manifiesta que existe un halo de contaminación en el suelo superficial del área por partículas minerales finas, lo que atribuye a la dispersión eólica de partículas desde la presa de jales. Asimismo, debe considerarse que existen reportes sobre el potencial riesgo para la salud de las poblaciones aledañas en la región de Zimapán, asociado a la concentración natural de As en agua para consumo humano (agua subterránea), con niveles que alcanzan hasta $1.0 \mathrm{mg} / \mathrm{L}$ (Armienta et al. 1997).

Como resultado de la actividad minera, se presentan diferentes problemas relacionados con la producción de polvo, ya sea durante el procesamiento del mineral o por la acumulación de sus residuos en presas de jales (Roy et al. 2012).

La dispersión de los jales mineros en forma de polvo provoca riesgos a la salud humana, ya que las partículas de menor tamaño que forman aerosoles (i.e., quedan suspendidas en el aire) pueden ser inhaladas $(<100 \mathrm{~mm})$. Cuando son torácicas $(<10$ $\mathrm{mm}$ ), pueden acumularse en el sistema respiratorio (incluyendo los pulmones) y producir daños físicos si son insolubles y toxicidad si se solubilizan; especialmente las partículas respirables $(<4 \mathrm{~mm})$ pueden pasar al torrente sanguíneo (INSHT 2008). El polvo generado por la acción del viento en las presas de jales donde se almacenan los residuos de la industria minera, representa un riesgo incluso después de que se hayan abandonado las actividades productivas en la mina (Puga et al. 2006b). Cuando se ingieren el proceso es completamente diferente, ya que pasan 
por el estómago y luego por el intestino donde la mayor parte es desechada, pero si son solubles una porción puede ser absorbida en las condiciones de este órgano.

Moreno-Tovar et al. (2012) argumentan, con relación a los jales de una mina en Zimapán, que existen evidencias de un cerco de suelo superficial contaminado en el área por partículas minerales finas, lo que atribuyen a la dispersión eólica de partículas desde la presa de jales.

Asimismo, se sabe que dependiendo de su forma química, los contaminantes pueden encontrarse como especies bioaccesibles, es decir, aquellas que tienen mayor potencial de migrar de una matriz a otra (Méndez y Maier 2008, Meza-Figueroa et al. 2009). Para tal efecto, el método de extracción basado en la fisiología (physiologically based extraction test, PBET) reportado por Ruby et al. (1996), así como otros procedimientos, simulan las condiciones del sistema gastrointestinal. Estos métodos in vitro determinan la bioaccesibilidad, ya que la biodisponibilidad se mide in vivo (MorenoTovar et al. 2012).
En este trabajo se determinó la bioaccesibilidad del As presente en jales del distrito minero de $\mathrm{Zi}$ mapán, Hidalgo, México, y se cuantificó la concentración de arsénico por tamaño de partícula desde 0.18 hasta $10 \mu \mathrm{m}$, lo cual incluye a las fracciones torácica y respirable, mediante una digestión ácida y por espectrometría de absorción atómica acoplada a generador de hidruros (EAA-GH).

\section{MATERIALES Y MÉTODOS}

\section{Descripción del área de estudio}

El estado de Hidalgo presenta una gran variedad de yacimientos, tanto metálicos (e.g., manganeso, plomo, plata, cobre, oro) como no metálicos (e.g., caliza, yeso, caolín, diatomita, piedra pómez, mármol, etc.). El desarrollo de la industria minero-metalúrgica comenzó hace aproximadamente 500 años (Labastida 2014).

En la región minera de Zimapán existe un gran número de yacimientos en explotación. En la figura 1 se presenta el panorama minero del estado de Hidalgo (SGM 2014). Las minas El Carrizal y El Monte están

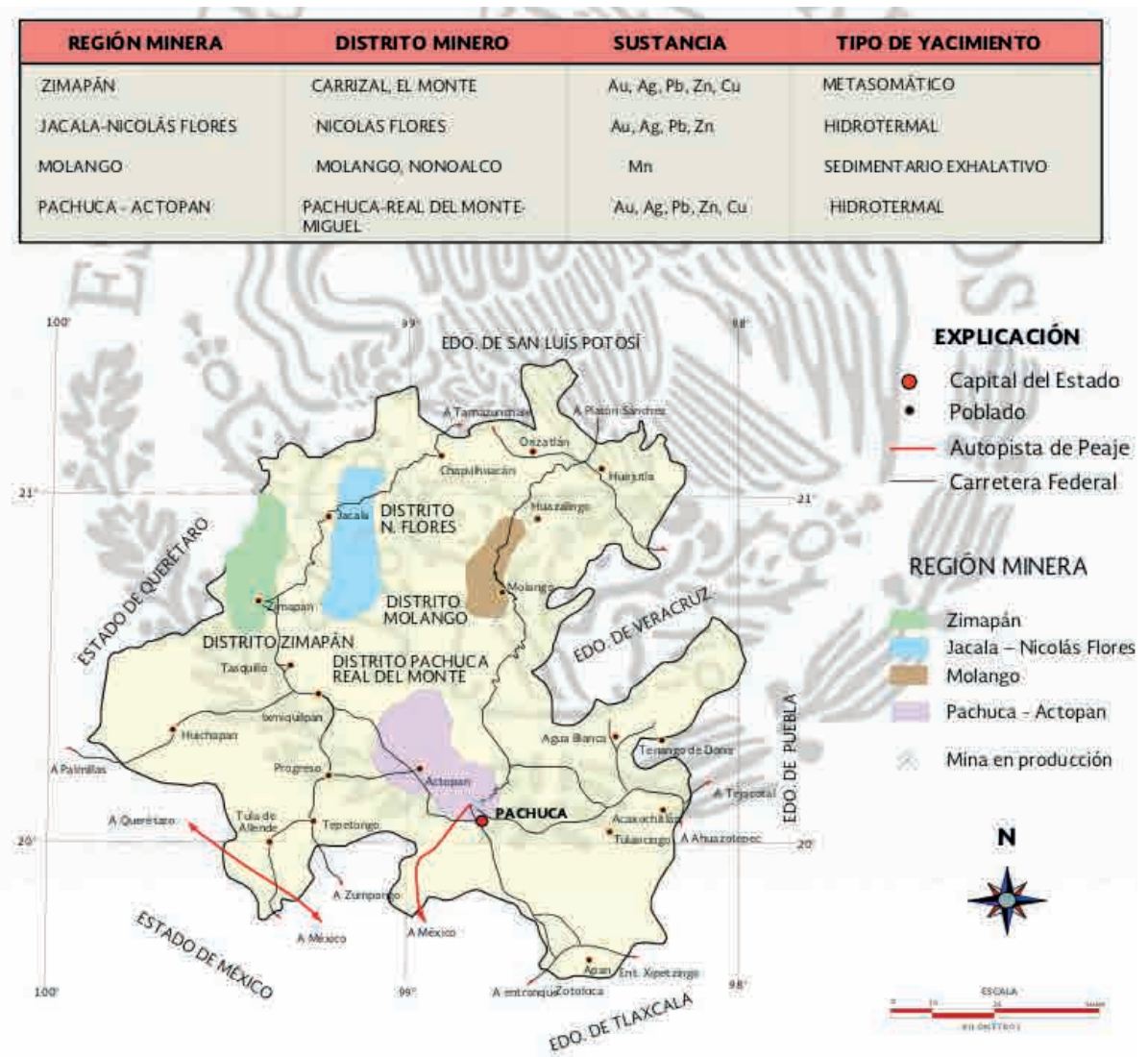

Fig. 1. Panorama minero del estado de Hidalgo (SGM 2014) 
constituidas por sulfuros y sulfosales: galena $(\mathrm{PbS})$, esfalerita $(\mathrm{ZnS})$, pirita $\left(\mathrm{FeS}_{2}\right)$, calcopirita $\left(\mathrm{CuFeS}_{2}\right)$, arsenopirita $\left(\mathrm{AsFeS}_{2}\right)$, pirrotita $\left(\mathrm{Fe}_{1-\mathrm{x}} \mathrm{S}\right)$, bornita $\left(\mathrm{Cu}_{5} \mathrm{FeS}_{4}\right)$, estibina $\left(\mathrm{Sb}_{2} \mathrm{~S}_{3}\right)$ y boulangerita $\left(\mathrm{Pb}_{5} \mathrm{Sb}_{4} \mathrm{~S}_{11}\right)$.

Respecto del uso de suelo, el Valle de Zimapán y el municipio de Nicolás Flores son dos de los escenarios más afectados por la extracción minera. Actualmente no se aplica alguna política ambiental que regule la mala disposición de materiales de desecho, que termina compactando el suelo y contribuyendo a su erosión. El Valle de Zimapán se considera una cuenca exorreica, es decir, una cuenca abierta con salidas en las vertientes sur y suroeste, lo cual permite la circulación de los vientos durante la mayoría del año. De abril a diciembre la velocidad promedio de los vientos es de 0.5 a $2.1 \mathrm{~m} / \mathrm{s}$. Los aspectos socioeconómicos de la zona de Zimapán destacan por ser una región económicamente estancada como resultado de las políticas urbanas a nivel nacional y estatal. Lo anterior, junto con los procesos de emigración, ha influido en la tasa de crecimiento poblacional, que ha registrado números negativos durante las últimas décadaso (CONABIO 2008).

\section{Muestreo}

Los trabajos de muestreo se realizaron en una instalación minera ubicada en el municipio de Zimapán (Fig. 1). Se tomaron muestras de jales mineros, los cuales son el producto de la trituración y molienda del mineral después de que se han recuperado los metales de interés a través de procesos físicos y químicos. Es importante aclarar que los jales mineros varían en su composición química y mineralógica, dependiendo de cada yacimiento (Medel et al. 2008).

Se decidió realizar un muestreo exploratorio para establecer la presencia de contaminación, basado en la norma NMX-AA-132-SCFI (SE 2006). La toma fue superficial en el perímetro de la presa 9, la cual se encuentra en operación, de manera que se trabajó con jales que provenían de procesamientos recientes.

El muestreo sobre la presa se realizó siguiendo una longitud de 1.0 a $1.5 \mathrm{~m}$ y a una profundidad de $30 \mathrm{~cm}$, con un peso aproximado por muestra de $1.5 \mathrm{~kg}$. La referencia del punto de muestreo se obtuvo mediante un posicionador geográfico satelital (GPS). Las muestras se manejaron según la norma mexicana para evitar alteraciones que pudieran ocurrir durante la recolección, transporte y manipulación.

El muestreo fue realizado por el personal de la mina, quienes tomaron siete muestras en diferentes puntos de la presa de jales 9, la cual tiene una extensión de poco menos de media hectárea (Fig. 2).
Las coordenadas de los puntos de muestreo se indican en el cuadro I.

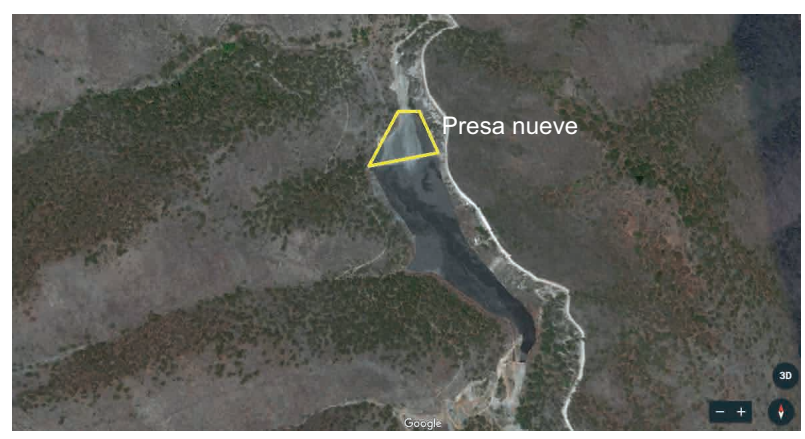

Fig. 2. Ubicación de la presa 9

CUADRO I. DATOS DEL MUESTREO REALIZADO

\begin{tabular}{lll}
\hline $\begin{array}{l}\text { Clave de la } \\
\text { muestra }\end{array}$ & Ubicación & Coordenadas \\
\hline HMC-1 & Cola de la presa nueve & $\begin{array}{l}20^{\circ} 49^{\prime}, 44.00^{\prime \prime} \mathrm{N} \\
99^{\circ} 22^{\prime} 20.40^{\prime \prime} \mathrm{O}\end{array}$ \\
\hline HMC-2 & Cabeza lado derecho & $20^{\circ} 49^{\prime}, 55.50^{\prime \prime} \mathrm{N}$ \\
& de la presa 9 & $99^{\circ} 22^{\prime} 27.53^{\prime \prime} \mathrm{O}$ \\
\hline HMC-3 & Mitad de la presa 9 & $20^{\circ} 49^{\prime} 51.03^{\prime \prime} \mathrm{N}$ \\
& & $99^{\circ} 22^{\prime} 27.53^{\prime \prime} \mathrm{O}$ \\
\hline HMC-4 & Cabeza del lado izquierdo & $20^{\circ} 49^{\prime} 54.711^{\prime \prime} \mathrm{N}$ \\
& de la presa 9 & $99^{\circ} 22^{\prime} 26.19^{\prime \prime} \mathrm{O}$ \\
\hline HMC-5 & Centro de la cabeza de & $20^{\circ} 49^{\prime} 54.77^{\prime \prime} \mathrm{N}$ \\
& la presa 9 & $99^{\circ} 22^{\prime} 28.82^{\prime \prime} \mathrm{O}$ \\
\hline HMC-6 & Borde de & $20^{\circ} 49^{\prime} 56.07^{\prime \prime} \mathrm{N}$ \\
& la presa 9 & $99^{\circ} 22^{\prime} 29.69^{\prime \prime} \mathrm{O}$ \\
\hline HMC-7 & Proceso de & $20^{\circ} 49^{\prime} 55.59^{\prime \prime} \mathrm{N}$ \\
& la presa 9 & $99^{\circ} 22^{\prime} 29.03^{\prime \prime} \mathrm{O}$ \\
\hline
\end{tabular}

\section{Análisis de la concentración total de metales Pretratamiento de las muestras}

Las muestras se sometieron a los procesos que se indican a continuación antes de la cuantificación del contenido de arsénico. Primero se realizó un secado a temperatura ambiente en un cuarto cerrado y libre de corrientes de aire, para evitar el contacto con éste y con el polvo, así como la contaminación de partículas de una muestra a otra. Para obtener estas condiciones las muestras se colocaron en bandejas de plástico y se registró su peso hasta obtener un peso constante, lo cual tomó aproximadamente $\sim 25$ días. La humedad final de las muestras fue menor al 
$1 \%$. Posteriormente se tamizaron y homogeneizaron; se tomaron porciones de aproximadamente $20 \mathrm{~g}$ de cada muestra para su análisis y la cantidad restante fue empacada en bolsas herméticas de polietileno e identificada para su resguardo

\section{Cuantificación de arsénico (As) total en las mues- tras obtenidas}

Para cuantificar el As se empleó la técnica de EAA-GH por el método USEPA 7061A (USEPA 1992). La cuantificación se realizó en un espectrofotómetro Perkin Elmer AAS 2380 equipado con un sistema de generador de hidruros MHS-10. Los parámetros utilizados para el análisis se muestran en el cuadro II. Todas las soluciones se prepararon con agua desionizada (18 $\mathrm{M} \Omega \times \mathrm{cm}$, Millipore Milli-Q). Se utilizó un estándar de alta pureza de As de $1000 \mu \mathrm{g} / \mathrm{mL}$. El As se almacenó a $4{ }^{\circ} \mathrm{C}$ y las diluciones se prepararon diariamente para cada análisis.

\section{CUADRO II. PARÁMETROS PARA LACUANTIFICACIÓN DE ARSÉNICO}

\section{Cuantificación de As}

Longitud de onda

Tiempo de integración

Intensidad de lámpara

Abertura (slit)

Tiempo de lectura

La digestión de las muestras se realizó tomando como referencia el método USEPA 3050B (USEPA 1996), el cual ayuda a determinar los elementos ambientalmente disponibles. Se utilizó una mezcla de ácido nítrico (Baker, Instra-analysed, 70.0 \%) y ácido clorhídrico (Baker, Instra-analysed, 36.5-38.0\%) en proporción 3 a 1.

\section{Determinación de la biodisponibilidad in vitro de arsénico}

La evaluación de la biodisponibilidad se realizó tomando como base la prueba PBET, la cual consiste en un sistema de prueba in vitro para predecir la liberación de metales en una matriz. Nuestro interés en realizar esta prueba radica en que la contaminación ambiental por polvo en la zona de beneficio se dispersa por el flujo de vehículos que transportan los materiales, exponiendo no sólo a los trabajadores en el sitio de extracción, sino también a las comunidades aledañas. Uno de los grupos poblacionales más expuestos son los menores de edad, quienes juegan diariamente en patios escolares y parques públicos y pueden ingerir el polvo de sus propias manos.

Esta prueba fue adaptada del método MGA 0291 "Disolución de fármacos en formas farmacéuticas sólidas", reportado en la Farmacopea de los Estados Unidos Mexicanos (SSA 2011). La prueba de disolución es un método que permite determinar la liberación de un principio activo en el medio de prueba, e implica el control de una serie de variables de origen diverso que afectan el patrón de flujo hidrodinámico en la interfaz sólido-líquido, y determinan la obtención de resultados reproducibles.

Una vez que se obtuvieron los resultados de la concentración de As total en cada muestra, se seleccionaron cinco muestras para la prueba de biodisponibilidad in vitro, las cuales presentaban las concentraciones más bajas y las más altas, siendo éstas las muestras 1, 2, 5, 6 y 7 .

Las muestras se colocaron en un equipo Vankel a $37{ }^{\circ} \mathrm{C}$ (Fig. 3) con seis vasos disolutores calibrados (exactitud $\pm 1 \%$ ) con $400 \mathrm{~mL}$ de una solución gástrica preparada según el método de Ruby et al. (1996), como se muestran en la figura 3. La extracción se dejó reposar durante 10 min; posteriormente se agitó a $100 \mathrm{rpm}$, velocidad que garantiza un patrón hidrodinámico para evitar turbulencias que alteren el tamaño de partícula. Como indica el método, la solución inicial se debe desgasificar para evitar la presencia de gases disueltos en el medio de disolución. Pasados los 10 min de agitación se tomó una alícuota de 20 $\mathrm{mL}$ utilizando una jeringa y se repuso el volumen con solución gástrica. Éste paso se repitió a los 40 y 60 min. Una vez que se tomó la última muestra, las soluciones de los digestores se llevaron a un

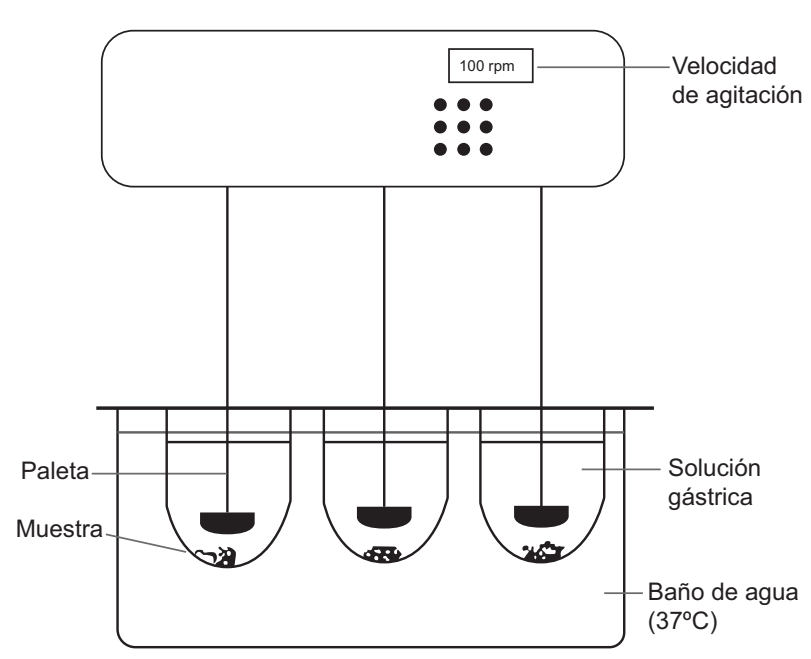

Fig. 3. Esquema del disolutor Vankel 
pH de 7 utilizando bicarbonato de sodio (polvo JT Baker QP) y se agregaron $700 \mathrm{mg}$ de sales biliares (Sigma-Aldrich grado microbiológico) y $200 \mathrm{mg}$ de pancreatina (Sigma-Aldrich grado USP) para emular las condiciones intestinales. Se tomaron dos alícuotas más a los 120 y 240 min. Todas las alícuotas se obtuvieron con filtros de nitrocelulosa de $0.45 \mu \mathrm{m}$ y se centrifugaron a $3500 \mathrm{rpm}$ durante $15 \mathrm{~min}$.

El líquido sobrenadante se colocó en matraces volumétricos de $50 \mathrm{~mL}$ que se llenaron hasta la marca de aforo. Posteriormente se colocó en envases de polietileno y se refrigeró a $4{ }^{\circ} \mathrm{C}$ para el posterior análisis de la concentración del As liberado por EAA-GH.

\section{Determinación de la concentración de arsénico por tamaño de partícula}

Durante esta etapa sólo se utilizaron dos muestras, HMC-1 y HMC-7, con apariencia de pequeñas partículas translúcidas, además de material grueso cuyo diámetro fue de 0.2 a $2.0 \mathrm{~cm}$. La masa utilizada en ambas muestras fue de 100.02 y $100.11 \mathrm{~g}$, respectivamente.

El experimento consistió en la generación de un aerosol que permitiera transportar la muestra sólida, intentando simular condiciones de levantamiento de polvos por medio de un equipo generador de turbulencias acoplado a un sistema de aire comprimido (Fig. 4). El aire comprimido funciona como gas acarreador acompañado de agitación magnética

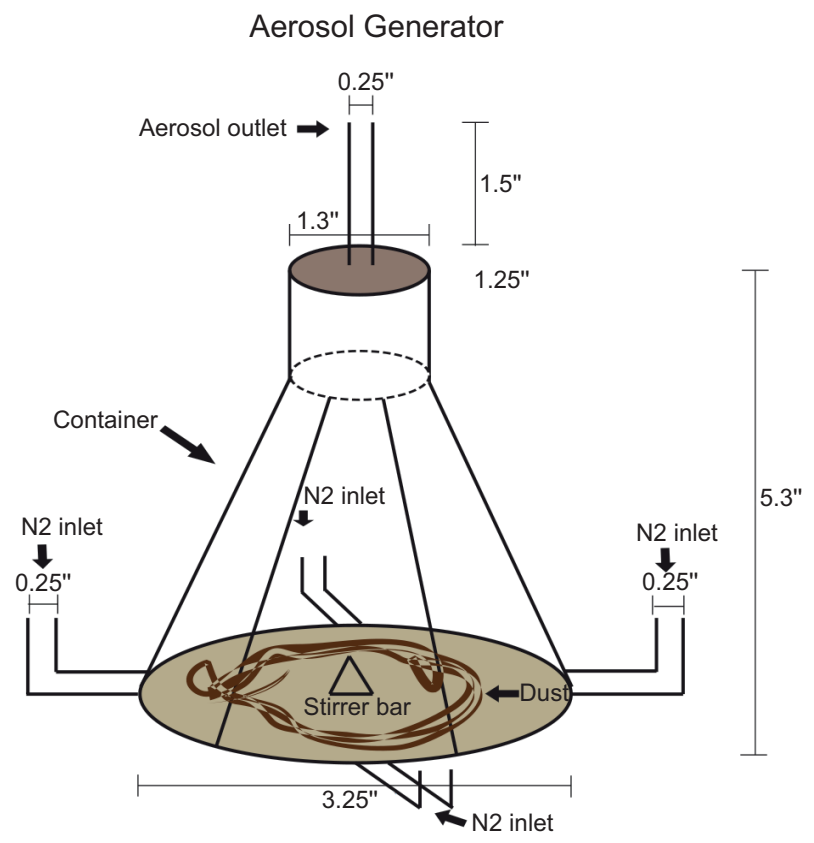

Fig. 4. Generador de aerosol en fase seca (Ladino y Abbatt 2013) para generar la suspensión en fase seca a partir de la muestra sólida. El equipo está construido con acero inoxidable para evitar interacciones electroestáticas entre sus paredes y las partículas en suspensión (Ladino y Abbatt 2013).

Después de haberse generado la suspensión, el siguiente paso fue la cuantificación de la concentración de partículas en el aerosol, que se formó con el objetivo de generar un flujo constante de partículas. La medición se realizó por medio del contador de partículas condensadas (CPC) modelo TSI 3010, el cual detecta y cuenta las partículas de aerosol con un tamaño mayor a $30 \mathrm{~nm}$. Esto es posible dado que las partículas aumentan su tamaño al exponerlas a un ambiente sobresaturado de butanol. Las partículas son detectadas por medios ópticos (láser/detector) y su concentración se expresa en número de partículas sobre centímetro cubico (\#partículas $/ \mathrm{cm}^{3}$ ).

$\mathrm{Al}$ sistema conjunto generador de aerosol-CPC se le acopló un sistema de dilución o un tanque de mezclado para remover las partículas mayores a 10 $\mathrm{mm}$ por medio de sedimentación por gravedad y para homogeneizar el aerosol. El tanque de dilución es de vidrio borosilicatado (Pyrex) y las conexiones se hicieron con manguera de teflón. Una vez que se tuvo acoplado el sistema de cuantificación de flujo se realizaron pruebas para indicar el nivel de agitación magnética que permitiera tener la mayor cantidad de partículas por el mayor tiempo posible, con la finalidad de asegurar que la masa colectada en la siguiente etapa del experimento fuese suficiente.

La tercera etapa fue la separación de las partículas de aerosol por tamaño, la cual se llevó a cabo con el instrumento Micro-Orifice Uniform Deposit Impactor (MOUDI) modelo 100R (Fig. 5). El impactador de cascada MOUDI permitió separar las partículas en ocho diferentes tamaños de diámetro $(0.18,0.32$, $0.56,1.0,1.8,3.2,5.6$ y $10 \mu \mathrm{m})$, acompañadas de un flujo de extracción de $30 \mathrm{~L} / \mathrm{min}$ reunido con recolectores de cuarzo durante aproximadamente $2 \mathrm{~h}$. Se realizaron dos pruebas con ambas muestras (HMC1 Y HMC7) por duplicado. Todas las muestras separadas por tamaño de partícula se analizaron mediante EAA-GH.

\section{Control de calidad}

Para calcular el límite de detección (LD) y el límite de cuantificación (LC) se realizaron tres curvas de calibración y se hizo una lectura de 10 blancos de muestra. Se obtuvo la desviación estándar de los mismos, además de la desviación estándar de la ordenada al origen y de la pendiente mediante un análisis de regresión lineal utilizando Excel. 


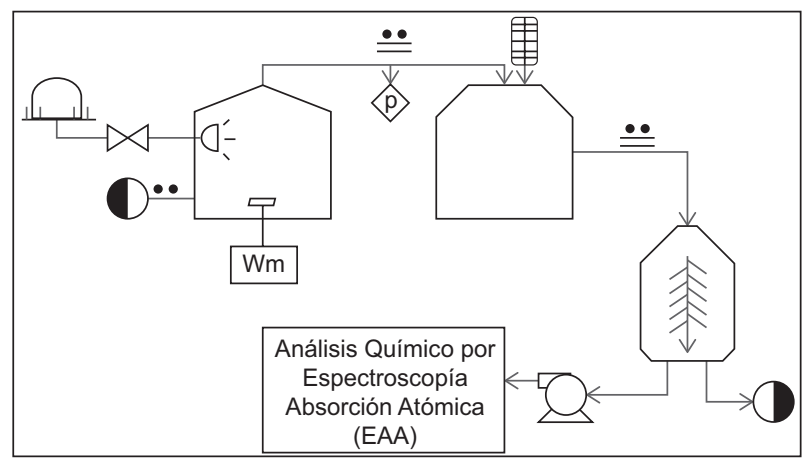

Fig. 5. Diagrama de flujo del proceso de separación de partículas en un aerosol a partir de su tamaño por medio del impactador MOUDI

Para evaluar el intervalo lineal se preparó una curva de calibración de 5 a 50 ng de arsénico y la sensibilidad se determinó mediante la pendiente de la curva de calibración. La exactitud del método se evaluó mediante el porcentaje de recobro, al cual se le adicionó una cantidad conocida de estándar de arsénico antes del proceso de digestión. Todas las muestras fueron analizadas por triplicado. Los resultados se expresan como el valor medio con la desviación estándar como medida de dispersión.

\section{RESULTADOS Y DISCUSIÓN}

Tal como se mencionó en la metodología, el muestreo realizado fue de tipo exploratorio y a una profundidad de $30 \mathrm{~cm}$, es decir, superficial. Los puntos de muestreo fueron elegidos de acuerdo con la descripción de un muestreo sistemático, puntualizado en la Norma Mexicana NMX-AA-132-SCFI-2006.

\section{Cuantificación de metales}

Los resultados de la determinación de la concentración total de As contenido en los jales arrojaron valores por encima de los límites establecidos en la NOM-147-SEMARNAT/SSA1-2004 (SEMARNAT 2007), la cual establece 260 ppm como límite máximo permisible para un suelo de uso industrial. En el cuadro III y la figura 6 se puede observar que las concentraciones en las siete muestras sobrepasan las 3000 ppm. Los principales estadísticos que describen el comportamiento de los niveles de As en las muestras estudiadas se indican en el cuadro IV.

La dispersión que presentan estos datos se indica en la figura $\mathbf{6}$, donde se observa que en todas las muestras la concentración está por encima de la distribución presentada; adicionalmente, existen
CUADRO III. CONCENTRACIONES TOTALES DE ARSÉ$\mathrm{NICO}$

\begin{tabular}{cc}
\hline Muestra & As (ppm) \\
\hline 1 & $3016.48 \pm 402.77$ \\
2 & $4026.49 \pm 420.06$ \\
3 & $4074.04 \pm 438.79$ \\
4 & $3516.24 \pm 555.99$ \\
5 & $4808.55 \pm 359.16$ \\
6 & $5469.26 \pm 984.81$ \\
7 & $8744.53 \pm 698.54$ \\
\hline
\end{tabular}

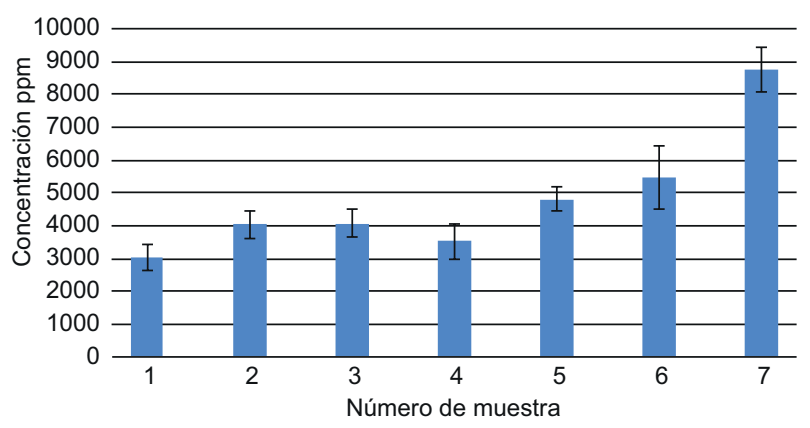

Fig. 6. Concentraciones totales de arsénico

valores (incluyendo el promedio) que se encuentran por arriba del valor establecido por la NOM-147-SEMARNAT/SSA1-2004 (SEMARNAT 2007), lo cual concuerda con estudios realizados de la misma zona que reportan concentraciones de As de 119 a 38700 $\mathrm{mg} / \mathrm{kg}$ (Moreno-Tovar et al. 2012).

\section{Determinación de la biodisponibilidad in vitro de arsénico}

En la figura 7 se observa que, de acuerdo con lo reportado en la literatura, el As tiene una limitada disolución en condiciones gastrointestinales (Ruby et al. 1999). La liberación de As ocurre predominantemente a $\mathrm{pH}$ ácido, esto es, en condiciones gástricas, por lo que el método aplicado indica que posiblemente la mayoría del arsénico ingerido sea excretado por el organismo. A pesar de que el As se encuentra presente en altas concentraciones en esta zona, su porcentaje de bioaccesibilidad in vitro es bajo. Este comportamiento parece normal para este tipo de muestras, ya que la principal fase portadora de As es la arsenopirita, tal como describen MorenoTovar et al. (2012) en su estudio, donde reportan un comportamiento similar al observado en el presente estudio, tanto en la fase gástrica como intestinal.

El valor obtenido de la biodisponibilidad in vitro es mayor al valor guía reportado por la organización 
CUADRO V. SEPARACIÓN DE PARTÍCULAS DE AEROSOL POR TAMAÑO CON IMPACTADOR DE CASCADA MOUDI

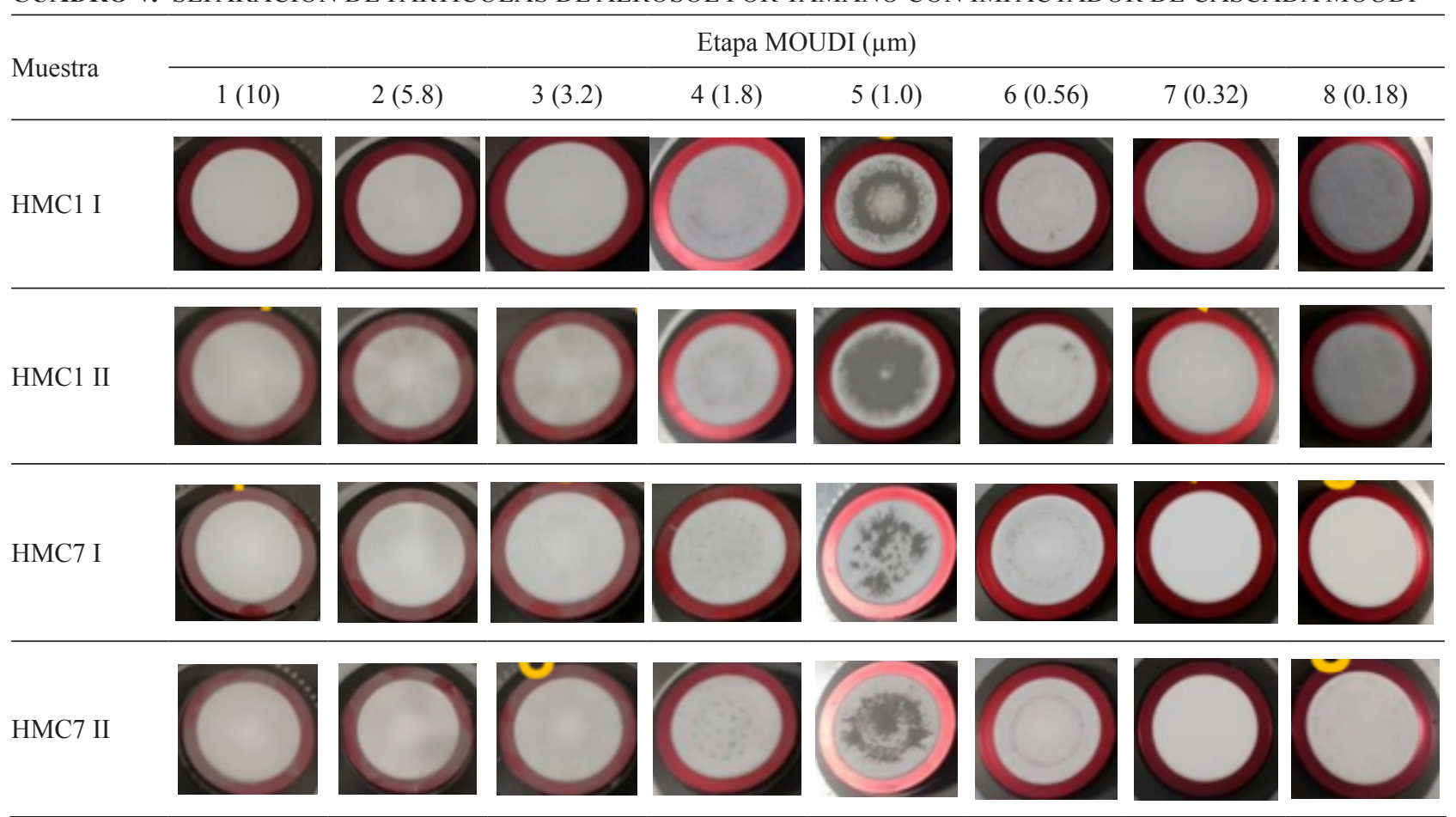

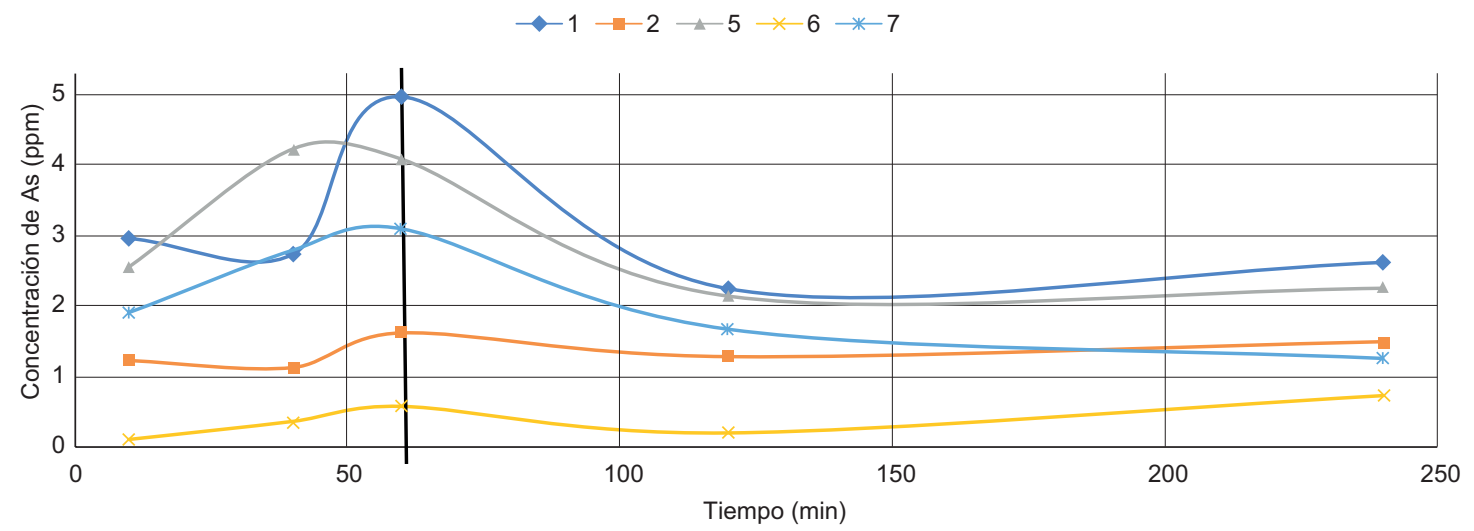

Fig. 7. Liberación de arsénico en fase gástrica e intestinal

mundial de la salud (OMS 2018b) para arsénico en agua potable $(10 \mu \mathrm{g} / \mathrm{L})$. Se debe tomar en cuenta que son matrices diferentes y los límites para consumo humano son mucho más estrictos. Consideramos que la ingesta de polvo de jales se da accidentalmente y no de manera recurrente.

Es importante señalar que para los estudios de biodisponibilidad in vitro es necesario tomar en cuenta que la naturaleza y composición geoquímica de la matriz representa un factor determinante en la fuerza de unión del metal; por lo tanto, en su liberación al medio. Así lo señala Moreno-Tovar (2006) en su estudio sobre las composiciones químicas y mineralógicas de jales dentro del mismo distrito de Zimapán, donde reporta que están constituidas principalmente por una mineralización metálica abundante (sulfuros, sulfosales, etc.) y por una no metálica formada por minerales calcosilicatados, calcita y cuarzo. Dichas mineralogías fueron confirmadas por los estudios de microscopia óptica y electrónica de barrido y por difracción de rayos $\mathrm{X}$.

Por otro lado, hay que recordar que la fracción biodisponible de una sustancia es aquella que alcanza la circulación sistémica y que, además, no es la 
misma, cuantitativamente hablando, que aquella que pudiera ser absorbida, sino menor.

A pesar de los resultados obtenidos, se debe hacer énfasis en la naturaleza in vitro de este estudio, lo que nos permite hacer ciertas predicciones del comportamiento del contaminante in vivo, más no aseveraciones. Se sabe que el comportamiento de ciertas sustancias en el organismo puede diferir en menor o mayor proporción del observado experimentalmente, ya que se trata de un sistema dinámico.

\section{Determinación de la concentración de arsénico por tamaño de partícula}

Al realizar diferentes combinaciones de agitación magnética y flujo de aire se encontró que un flujo de aire constante de aproximadamente $3 \mathrm{~L} / \mathrm{min}$ y el nivel 8 de agitación permiten mantener un flujo de partículas constante por el mayor tiempo posible, por lo que se establecieron como la condiciones para la generación del aerosol.

Se observó que la presión de aire y la agitación magnética son de suma importancia, ya que permiten definir la cantidad de muestra sólida que será aerolizada. Sin embargo, estos parámetros resultaron ser específicos para las características del suelo donde se recolecte la muestra. Esta observación surgió debido a que en las pruebas preliminares se utilizó un tipo de suelo con diferentes características a las de las muestras de los jales, ya a que la cantidad de muestra era limitada (i.e., $100 \mathrm{~g}$ ).

Los resultados obtenidos durante la separación y recolección de partículas de aerosol por tamaño con el MOUDI se muestran en el cuadro V. Se observa que fue en la etapa 5 del recolector de partículas cuando se reunió la mayor cantidad de muestra. Esta etapa corresponde a un tamaño de partículas de $1 \mu \mathrm{m}$.

En la última parte del experimento se llevó a cabo el análisis químico por medio de EAA, con el fin de determinar la concentración de arsénico. Los resultados arrojados por esta técnica se determinaron en $\mathrm{mg} / \mathrm{kg}$ y se graficaron en función del tamaño de

CUADRO IV. ESTADÍSTICOS DESCRIPTIVOS PARA LA CONCENTRACIÓN DE ARSÉNICO

\begin{tabular}{lc}
\hline & As $(\mathrm{mg} / \mathrm{kg})$ \\
\hline Máximo & 9515.54 \\
Media & 4807.94 \\
Mediana & 4438.24 \\
Mínimo & 2731.76 \\
Desviación estándar & 1880.66 \\
Nivel de confianza $(95.0 \%)$ & 9515.54 \\
\hline
\end{tabular}

partícula para observar la relación que guardan estas variables (Fig. 8). Se pudo observar que la mayor concentración de partículas se encuentra en las etapas centrales, la cuales corresponden al rango de tamaños de partícula de 1.8 a $0.56 \mu \mathrm{m}$. Lo anterior da una idea del tipo de partículas que conforman la muestra, las cuales en este caso corresponden a los modos de acumulación y en la distribución de tamaño (Lohmann et al. 2016).

Cuando se analizan las etapas en que se presentó la mayor recolección de muestras, se observa específicamente que la mayor concentración de partículas ocurrió en la etapa 5, que corresponde a partículas de $1.0 \mu \mathrm{m}$, de modo que esta muestra pertenece al grupo de partículas $\mathrm{PM}_{2.5}$, siempre y cuando estas partículas sean emitidas al aire. Teniendo esto en cuenta, este tipo de partículas tiene un efecto directo sobre el cuerpo, ya que por su tamaño suelen alojarse en la región traqueobronquial del sistema respiratorio, pudiendo ocasionar un proceso apoptótico en las células (Chirino et al. 2015). Estudios previos sobre la bioaccesibilidad pulmonar del As por ingestión han demostrado que este metal puede causar cáncer de pulmón, entre otros padecimientos (Torres 2011, Ávila y Bovi 2018). Determinar la bioaccesibilidad pulmonar del As respirable es mucho más complejo. Dado que las partículas submicrométricas son las que pueden llegar eficientemente a los pulmones y solo las nanométricas pueden llegar a los alveolos e incluso al torrente sanguíneo (Wallace y Hobbs 2006), un análisis de bioaccesibilidad pulmonar del As respirable sería útil si altas concentraciones de este elemento se observaran en partículas nanométricas. Sin embargo, el presente estudio sugiere que éste no es el caso, por lo cual no se realizó un estudio de bioaccesibilidad pulmonar del As. Adicionalmente, como la masa de las partículas nanométricas es muy pequeña, la metodología utilizada en este estudio no permitiría realizar un estudio cuantitativo de bioaccesibilidad pulmonar del As.

\section{CONCLUSIONES}

De los puntos de muestreo seleccionados con base en los antecedentes históricos de la minería en el estado de Hidalgo, se observa que, en efecto, en el sitio se encontraron muestras con alta concentración de As, por lo que se asume que la metodología de selección y muestreo fue el adecuado para cumplir con los objetivos de este estudio. Las muestras sobrepasan los límites establecidos por la NOM-147-SEMARNAT/ SSA1-2004 (SEMARNAT 2007) para arsénico total, 


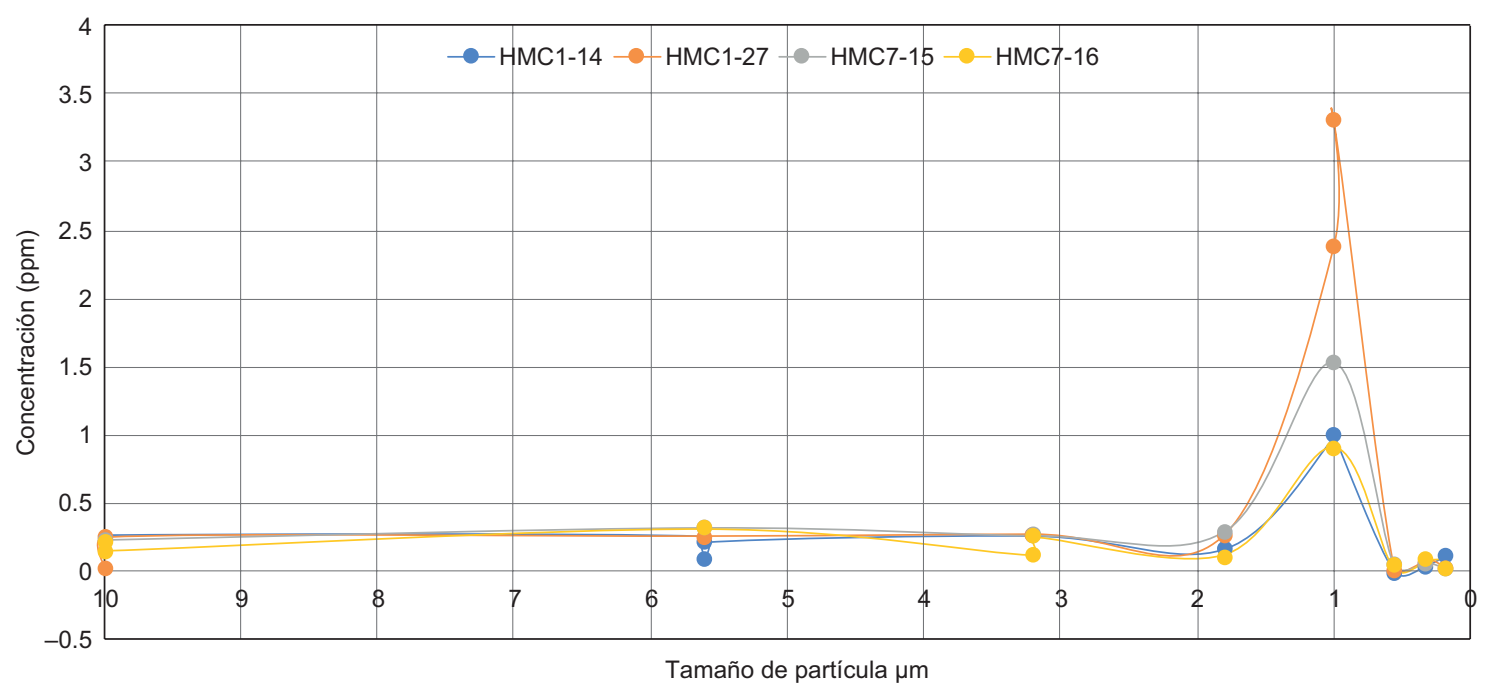

Fig. 8. Concentración de arsénico por tamaño de partícula

por lo que el ensayo de biodisponibilidad in vitro fue de gran utilidad aunado a la evaluación del material particulado, como una base para establecer el riesgo potencial al que está expuesta lo población cercana al sitio en estudio.

Los resultados muestran que el As se libera en mayor proporción cuando el $\mathrm{pH}$ del medio es ácido, esto es, en condiciones gástricas, existiendo una disminución cuando los niveles de $\mathrm{pH}$ tienden a la neutralidad en condiciones intestinales. Las cantidades de As registradas a lo largo de este ensayo representan la fracción bioaccesible, lo que al observar los valores obtenidos sugiere que la fracción biodisponible de dicho metal no alcanza las concentraciones necesarias para la aparición de efectos tóxicos que pongan en riesgo la salud humana.

En lo que se refiere a los resultados de la generación de un aerosol proveniente de residuos mineros, se observa que el sistema diseñado simula exitosamente el levantamiento de polvos por fenómenos atmosféricos a microescala. Los resultados muestran que la mayor cantidad de partículas corresponde a un tamaño de 0.56 a $1.8 \mu \mathrm{m}$, siendo las de $1.0 \mu \mathrm{m}$ las más abundantes; asimismo, la mayor concentración de As se presentó en partículas de este tamaño.

Este comportamiento se puede asociar a dos motivos: 1) que el metaloide se almacene en partículas ya existentes en este tamaño o 2) por procesos de cohesión y coagulación de partículas más pequeñas con arsénico almacenado.

Los resultados del estudio aportan información que coadyuva a determinar el riesgo potencial que representan los jales mineros para la población expuesta a la ingestión de polvos, así como la bioaccesibilidad como indicador de la biodisponibilidad (in vitro) de los elementos potencialmente tóxicos (EPT) contenidos en muestras de jales procedentes de la minería, con el fin de relacionar la bioaccesibilidad con la mineralogía de los residuos.

Finalmente, se puede recomendar la realización de una evaluación estacional (temporadas de lluvia y periodo de secas) que permita establecer la movilidad del metal y su compartimiento ambiental.

\section{AGRADECIMIENTOS}

Los autores agradecen a la Dirección General de Asuntos del Personal Académico (DGAPA), por el apoyo económico para el proyecto PAPIIT IA108417.

\section{REFERENCIAS}

Armienta M.A., Rodríguez R. y Cruz O. (1997). Arsenic content in hair of people exposed to natural arsenic polluted groundwater at Zimapán, Mexico. Bull. Environ. Contam. Toxicol. 59, 583-589. DOI: 10.1007/ s001289900519

Ávila N.M.E. y Bovi M.G. (2018). Bioaccesibilidad de arsénico y cálculos de ingestas en dietas consumidas por una población endémica de Taco Pozo. Comparación entre las ingestas infantiles y adultas. Rev. Toxicol. 35, 11-17.

Bartkowiak A. y Lemanowicz J. (2014). Application of biochemical tests to evaluate the pollution of the 
Unislaw Basin soils with heavy metals. Int. J. Environ. Res. 8 (1), 93-100. DOI: 10.22059/IJER.2014.698

Botín J.A. (2010). Integrating sustainability down to the operational levels of a mining company. Dyna 77 (161), 43-49.

Chirino Y.I., Sánchez-Pérez Y., Osornio-Vargas Á.R., Rosas I. y García-Cuéllar C.M. (2015). Sampling and composition of airborne particulate matter $\left(\mathrm{PM}_{10}\right)$ from two locations of Mexico City. Toxicol. Lett. 4, 353-356. DOI: 10.1016/j.dib.2015.06.017

CONABIO (2008). Informe final del proyecto DQ006. Ordenamiento ecológico territorial regional en los municipios donde se ubica el Parque Nacional Los Mármoles. Hidalgo, México. Comisión Nacional para el Conocimiento y Uso de la Biodiversidad [en línea]. http://www.conabio.gob.mx/institucion/proyectos/ resultados/InfDQ006_1a_parte.pdf 06/02/2019

INEGI (2018). Producto interno bruto a precios corrientes. Cifras durante el tercer trimestre de 2018. Instituto Nacional de Estadística y Geografía [en línea]. http:// www.beta.inegi.org. $\mathrm{mx} /$ contenidos/saladeprensa/ notasinformativas/2018/pib_precr/pib_precr2018_05. pdf 06/02/2019

INSHT (2008). Notas técnicas de prevención 808. Instituto Nacional de Seguridad e Higiene en el Trabajo [en línea]. http://www.insht.es/InshtWeb/ Contenidos/Documentacion/FichasTecnicas/NTP/ Ficheros/786a820/808\%20web.pdf 23/01/2019

Labastida I. (2014). Evaluación de un sistema basado en rocas calizas, para tratar el drenaje ácido de mina en distrito minero de Zimapán, Hidalgo. Tesis de Doctorado. Posgrado en Ciencias de la Tierra, Universidad Nacional Autónoma de México, Ciudad de México, México, 87 pp.

Ladino L.A. y J.P.D. Abbatt. (2013). Laboratory investigation of Martian water ice cloud formation using dust aerosol simulants. J. Geophyis. Res. 118, 14-25. DOI: 10.1029/2012JE004238

Lillo J. (2011). Impactos de la minería en el medio natural. Grupo de Geología. Grupo de Estudios en Minería y Medioambiente. Universidad Rey Juan Carlos [en línea].

https://reformaminera.wordpress.com/2008/06/02/34-impactos-de-la-mineria-en-el-medio-natural/23/01/2019

Lohmann U., Lüönd F. y Mahrt F. (2016). An introduction to clouds: From the microscale to climate. Cambridge University Press, Cambridge, Reino Unido, 81 pp.

Martínez N.V., Turrubiarte K.L. y Flores L.M. (2000). Comportamiento químico de $\mathrm{Pb}$ y $\mathrm{Zn}$ en suelos aledaños a una presa de jales. Memorias. XXXV Congreso Mexicano de Química de la Sociedad Química de México, San Luis Potosí, S.L.P., 24 al 28 de septiembre, CD-ROM.
Medel A., Ramos S., González-Avelar F., Godínez L. y Rodríguez F. (2008). Caracterización de jales mineros y evaluación de su peligrosidad con base en su potencial de lixiviación. Conciencia Tecnológica 35, 32- 35.

Méndez M.O. y Maier R.M. (2008). Phytoremediation of mine tailings in temperate and arid environments. Rev. Environ. Sci. Biotechnol. 7 (1), 47-59. DOI: 10.1007/ s11157-007-9125-4

Meza-Figueroa D., Maier R.M., de la O-Villanueva M., Gómez-Álvarez A., Moreno-Zazueta A., Rivera J. y Palafox-Reyes J. (2009). The impact of unconfined mine tailings in residential areas from a mining town in a semi-arid environment: Nacozari, Sonora, Mexico. Chemosphere 77 (1), 140-147. DOI: 10.1016/j.chemosphere.2009.04.068

Moreno-Tovar R. (2006). Evaluación geoquímicoambiental de residuos (jales) de mineralizaciones poliméricas del estado de Hidalgo, México. Tesis de Dctorado. Universidad Autónoma de San Luis Potosí, San Luis Potosí, México, 197 pp.

Moreno-Tovar R., Téllez-Hernández J. y Monroy-Fernández M. G. (2012). Influencia de los minerales de los jales en la bioaccesibilidad de arsénico, plomo, zinc y cadmio en el distrito minero Zimapán, México. Rev. Int. Contam. Ambie. 28 (3), 203-218.

OMS (2018b). Arsénico. Organización Mundial de la Salud [en línea]. http://www.who.int/es/news-room/ fact-sheets/detail/arsenic 18/01/2019

Pérez-Martínez I. y Martín-Romero F. (2015). Uso de parámetros indirectos para la evaluación de la contaminación de suelos por metales pesados en una zona minera de San Luis Potosí, México. B. Soc. Geol. Mex. 67 (1), 1-12.

Puga S., Sosa M., Lebgue T., Quintana C. y Campos A. (2006a). Contaminación por metales pesados en suelo provocada por la industria minera (Heavy metals pollution in soils damaged by mining industry). Ecología Aplicada 5, 149-155.

Puga S., Sosa M., De la Mora A., Pinedo C. y Jiménez J. (2006b). Concentraciones de As y $\mathrm{Zn}$ en vegetación nativa cercana a una presa de jales. Rev. Int. Contam. Ambie. 22 (2), 75-82.

Romero F.M., Armienta M.A., Gutiérrez M.E. y Villaseñor G. (2008). Factores geológicos y climáticos que determinan la peligrosidad y el impacto ambiental de jales mineros. Rev. Int. Contam. Ambie. 24 (2), 43-54.

Roy P., Kumar-Sikdar P., Singh G. y Kumar-Pal A. (2012). Source apportionment of ambient $\mathrm{PM}_{10}$ : A case study from a mining belt of Orissa. Atmósfera 25 (3), 311-324.

Ruby M.V., Davis A., Schoof R., Eberle S. y Sellstone C.M. (1996). Estimation of lead and arsenic bioavailability using a physiologically based extraction test. Environ. Sci. Technol. 30 (2), 422-430. DOI: 10.1021/ es950057z 
Ruby M.V., Schoof R., Brattin, W., Goldade M., Post G., Harnois M., Mosby D.E., Casteel S. W., Berti W., Carpenter M., Edwards D. Cragin D. y Chappell W. (1999). Advances in evaluating the oral bioavailability of inorganics in soil for use in human health risk assessment. Environ. Sci. Technol. 33 (21), 3697-3705. DOI: $10.1021 / \mathrm{es} 990479 \mathrm{z}$

SE (2006). Norma Oficial Mexicana NMX-AA-132-SCFI. Muestreo de suelo para la identificación y la cuantificación de metales y metaloides, y manejo de la muestra. Secretaría de Economía. Diario Oficial de la Federación, 5 de septiembre.

SGM (2016). Anuario estadístico de la minería mexicana 2016, edición 2017. Servicio Geológico Mexicano, Secretaría de Energía [en línea].

https://www.sgm.gob.mx/productos/pdf/Anuario_2016_ Edicion_2017.pdf 14/01/2019

SGM (2014). Panorama minero del Estado de Hidalgo. Servicio Geológico Mexicano, Secretaría de Energía [en línea]. http://www.sgm.gob.mx/pdfs/HIDALGO. pdf 14/01/2019

SEMARNAT (2007). Norma Oficial Mexicana NOM147-SEMARNAT/SSA1-2004. Que establece criterios para determinar las concentraciones de remediación de suelos contaminados por arsénico, bario, berilio, cadmio, cromo hexavalente, mercurio, níquel, plata, plomo, selenio, talio y/o vanadio. Secretaría de Medio Ambiente, Recursos Naturales y Pesca. Diario Oficial de la Federación, 26 de abril.

SSA (2011). Métodos generales de análisis: Método disolución MGA 0291. En: Farmacopea de los Estados Unidos Mexicanos. Tomo 1. 10a ed. Secretaría de Salud, México, pp. 303-310.

Torres S. (2011). Bioaccesibilidad de arsénico y mercurio en alimentos con potencial riesgo toxicológico. Tesis de Doctorado. Departamento de Química Analítica, Universitat de Valencia, España, 42 pp.

USEPA (1992). Method 7061A: Arsenic (Atomic absorption, gaseous hydride). Revision 1. Washington, DC, EUA, 6 pp.

USEPA (1996). Method 3050B: Acid digestion of sediments, sludges, and soils. Revision 2. Washington, DC, EUA, 12 pp.

Wallace J.M. y Hobbs P.V. (2006). Atmospheric science: An introductory survey. 2a ed. University of Washington. Elsevier, Burlington, EUA, 483 pp. 